\title{
Densidad, estructura de tallas y actividad reproductiva del caracol rosado Eustrombus gigas (Mesogastropoda: Strombidae) en Banco Chinchorro, México
}

\author{
Yuself R. Cala ${ }^{1,2}$, Alberto de Jesús Navarrete ${ }^{3}$, Frank A. Ocaña ${ }^{2,4}$ \& José Oliva Rivera ${ }^{3}$ \\ 1. Parque Nacional Desembarco del Granma. Belic s/n, Niquero, Granma, Cuba. \\ 2. Programa de doctorado en Ecología y Desarrollo Sustentable, El Colegio de la Frontera Sur, Unidad Chetumal. Ave. \\ Centenario, Km. 5.5, Col. Antorchista. Chetumal. Quintana Roo, México; sgigas21824@gmail.com \\ 3. Departamento de Aprovechamiento y Manejo de Recursos Acuáticos, El Colegio de la Frontera Sur (ECOSUR), \\ Unidad Chetumal, México; anavarre@ecosur.mx, joliva@ecosur.mx \\ 4. Centro de Investigaciones y Servicios Ambientales y Tecnológicos (CISAT), Holguín, Cuba; frankocisat@gmail.com
}

\section{Recibido 06-XI-2012. Corregido 08-III-2013. Aceptado 24-IV-2013.}

\begin{abstract}
Density, size structure and reproductive activity of the pink conch Eustrombus gigas (Mesogastropoda: Strombidae) in Banco Chinchorro, Mexico. The pink conch Eustrombus gigas is an important fisheries resource. At the regional level in the Caribbean, over-exploitation and habitat destruction have caused a decrease in the abundance of this resource. In order to provide necessary information for the species management in Mexico, this work aimed to analyze the total density, adult density, size structure and reproductive behavior of pink conch population at Banco Chinchorro during 2009-2010. Data from three seasons were obtained (rainy, dry and cold fronts periods) in three areas: Norte (North), Centro (Center) and Sur (South). The organisms were separated into two groups: (a) the criteria based upon legal harvest in Mexico: legal size conchs (siphonal length $\geq 200 \mathrm{~mm}$ ) and illegal size conchs (siphonal length $<200 \mathrm{~mm}$ ), and (b) the criteria based upon sexual maturity using the $15 \mathrm{~mm}$ lip thickness standard: lip $<15 \mathrm{~mm}$ as juvenile conch and lip $\geq 15 \mathrm{~mm}$ as adult conch. Copulation, spawning, egg masses and aggregations were evaluated as reproductive evidences. The highest total density was observed during the dry season with 384ind./ha, and the lowest during the rainy season with 127ind./ha. The highest density was reported at Sur (385ind./ha) and the lowest at Norte (198ind./ ha). The highest adult density was observed during the rainy season (8.33ind./ha), and the lowest occurred in the dry season (6.11ind./ha). Adult density values were 5.55, 7.05 and 8.33ind./ha for Centro, Sur and Norte areas, respectively. Adult densities were lower than the threshold needed for reproduction, and $42 \%$ of the population may be vulnerable to fishing, as they had the minimum size for catch (Lsi 200mm). Furthermore, only $2.2 \%$ of the population reached a Gl $>15 \mathrm{~mm}$ as sexual maturity indicator. During the study period, only six evidences of reproductive activity were observed. The smaller densities reported at Banco Chinchorro may cause reproduction events to be almost absent which in turn is sufficient evidence to show that the Allee Effect is acting on the queen conch population and there is an urgent need of fishery closure. Three important points were proposed for management of queen conch at Banco Chinchorro: total closure of fishing, systematic assessment of the conch population and the implementation of conch fishing refuge. Rev. Biol. Trop. 61 (4): 1657-1669. Epub 2013 December 01.
\end{abstract}

Key words: Allee effect, overfishing, reproduction, protected areas, Eustrombus gigas.

Los moluscos son uno de los grupos de invertebrados más diversos (Vokes \& Vokes, 1983). Los gasterópodos son considerados entre los más llamativos y poseen especies críticas en ambientes marinos poco profundos debido a su gran importancia económica (De
Jesús-Navarrete \& Oliva-Rivera, 1997). La subclase Archaeogastropoda, el segundo grupo más primitivo de los moluscos gasterópodos, apareció por primera vez en el período Cámbrico Inferior y dentro de ellos, el orden Mesogastropoda posee especies de gran tamaño, 
sobre todo los que pertenecen a la familia Strombidae. Estos caracoles se caracterizan por presentar una gran concha en espiral con un opérculo en forma de una garra (Randall, 1964; Alcolado, 1976).

La familia Strombidae cuenta con 136 especies distribuidas en todo el mundo y seis especies distribuidas en el Atlántico Tropical Occidental. Todas las especies están ampliamente distribuidas, pero una se destaca por su gran tamaño y su importancia en las pesquerías tropicales, la concha reina o caracol rosado (Eustrombus gigas Linnaeus 1758) anteriormente conocido como Strombus gigas (Petuch, 2004; Petuch \& Roberts, 2007).

En la actualidad E. gigas se puede encontrar en las Bermudas, las Bahamas, el sureste de Florida (incluyendo los Cayos de la Florida), Antillas Mayores y Menores, y las costas caribeñas de América Central y América del Sur al sur de Brasil (Abbott, 1974; Brownell \& Stevely, 1981; De Jesús-Navarrete \& Oliva-Rivera, 1997). La especie es uno de los recursos pesqueros costeros más valiosos en la región del Caribe (De Jesús-Navarrete \& Oliva-Rivera, 1997; Theile, 2001) y constituye un molusco con un elevado valor estético y ecológico. Según Appeldoorn (1994a), desde el punto de vista económico representa uno de los recursos costeros más importante para la región y ha sido considerado el segundo en importancia después de la langosta espinosa (Panulirus argus Latreille 1804) (De Jesús-Navarrete \& Oliva-Rivera, 1997). Debido al estado actual precario de sus poblaciones, la especie ha sido incluida desde 1992 en el apéndice II de la Convención sobre el Comercio de Especies Amenazadas de Fauna y Flora Silvestres (CITES) (Stoner, Sandt \& Boidron-Metairon, 1992; Stoner, Ray, Glazer \& McCarthy, 1996) y en la Lista Roja de Animales Amenazados de la Unión Internacional para la Conservación de la Naturaleza -UICN 1994- como especie 'comercialmente amenazada' (Gómez-Campo, Rueda \& García-Valencia, 2010).

En el Caribe, las investigaciones sobre esta especie son numerosas (Randall, 1964; Appeldoorn, 1990; Glazer \& Berg, 1992; Stoner et al. 1996; Basurto-Origel, Cruz-Domínguez, Martínez-Vázquez \& Cadena-Romero, 2000; De Jesús-Navarrete, 2001; De Jesús-Navarrete, Medina-Quej \& Oliva-Rivera, 2003; Jared, Taylor, Roy \& Hellberg 2006; Ávila-Poveda \& Baqueiro-Cárdenas, 2006; Gómez-Campo et al. 2010; Chávez-Villegas, Enríquez-Díaz, Cid-Becerra \& Aldana-Aranda, 2012; Peel \& Aldana-Aranda, 2012; Stoner, Davis, \& Booker, 2012a). En México, algunas de las áreas de pesca históricamente importantes de caracol rosado han sido clausuradas debido al agotamiento de los planteles y a la sobrepesca (Basurto-Origel et al. 2000). Actualmente, la pesca legal de caracol rosado está restringida al Banco Chinchorro, Estado de Quintana Roo (Basurto-Origel et al. 2000). Recientemente se ha propuesto una clausura indefinida de la pesquería de caracol rosado para todo el Estado de Quintana Roo, debido a las inquietudes manifestadas por los pescadores locales. Esto significaría de hecho una clausura total de su pesquería legal en México.

En Banco Chinchorro los estudios realizados con esta especie son también numerosos y abarcan varios temas: distribución, abundancia, crecimiento y mortalidad (De Jesús-Navarrete \& Valencia-Beltrán, 2003; De Jesús-Navarrete et al. 2003; Medina-Quej, Domínguez-Viveros \& De Jesús-Navarrete, 2005); evaluaciones con fines pesqueros (Basurto-Origel, Cadena, Escobedo \& Fernández, 2005; BasurtoOrigel, Cadena, Escobedo \& Figueroa, 2007; Basurto-Origel, Espinosa, Cob, Escobedo \& Figueroa, 2009; Basurto-Origel, Espinosa, Cob \& Escobedo, 2010; Basurto-Origel, Cervera \& Medina, 2011); distribución de larvas (De Jesús-Navarrete, 1999; Paris, Aldana-Aranda, Pérez-Pérez \& Kool, 2008); estudios genéticos (Pérez-Enríquez, García-Rodríguez, MendozaCarrión \& Padilla, 2011) y reproducción de la especie (Aldana-Aranda, Baqueiro-Cárdenas, Martínez-Morales, \& Ochoa-Báez, 2003). Sin embargo, los estudios que analizan no solo la densidad y estructura de edades de la población sino su relación con la frecuencia de eventos reproductivos, son realmente escasos. En el Caribe, se cuenta con los estudios de Egan 
(1985), Appeldoorn (1993) y Aldana-Aranda et al. (2003).

Stoner \& Ray (2000) en Bahamas, encontraron una relación estrecha entre la densidad del molusco y la frecuencia de evidencias reproductivas (apareamiento, masas ovígeras, entre otras), sin observar éstas cuando las densidades se encontraban por debajo de 56 adultos (ind./ha). Recientemente Stoner et al. (2012a) estimaron densidades similares (47 y 64ind adultos/ha en Islas Berry e Isla Andros en Bahamas, respectivamente) como umbral inferior para el encuentro de pareja. En Banco Chinchorro, México, De Jesús Navarrete et al. (2003) reportan muy poca evidencia reproductiva, también relacionada con la baja densidad de adultos a causa de la sobrepesca y hacen referencia a la posible ocurrencia del efecto Allee en la población.

Aquí se presenta un análisis sobre la densidad total y de adultos, estructura de tallas y la evidencia reproductiva de E. gigas en Banco Chinchorro, con el objetivo de brindar información necesaria para el manejo de la especie en México. Los resultados contribuirán a la actualización del plan de manejo de la Reserva de Biosfera Banco Chinchorro con elementos relevantes sobre la reproducción de E. gigas en el área.

\section{MATERIALES Y MÉTODOS}

Área de estudio: La Reserva de la Biosfera Banco Chinchorro (18 $36^{\prime} 12^{\prime \prime} \mathrm{N}-87^{\circ} 18^{\prime} 28^{\prime \prime}$ $\mathrm{W})$ es un arrecife en forma de atolón, que se encuentra ubicado en el Sistema Arrecifal Mesoamericano dentro de la Zona Económica Exclusiva de México y es uno de los más grandes de su tipo en la cuenca del Caribe (De Jesús Navarrete et al. 2003; Vázquez-Yeomans, Ordóñez-López, Quintal-Lizama, \& OrnelasRoa, 2003). Tiene una superficie de 144 360ha, e incluyen formaciones de arrecifes, lagunas, y tres cayos: Lobos, Centro y Norte, así como aguas oceánicas adyacentes (Fig. 1) (INESEMARNAP, 2000).

La profundidad del área de estudio varía de $12 \mathrm{~m}$ en la región sur a $2 \mathrm{~m}$ en el norte, y $3-7 \mathrm{~m}$ en la parte central y está cubierta de pastos marinos, principalmente Thalassia testudinum, Syringodium filiforme y algunas algas marinas (De Jesús-Navarrete et al. 2003).

Muestreo: Los muestreos fueron realizados durante el año 2009 y 2010 en tres zonas: Norte (17 245ha), Centro (21 110ha) y Sur (15 461ha), en 15 localidades de muestreo (cinco localidades por zona) consideradas tradicionalmente como zonas de pesca por los pescadores locales de E. gigas. En cada localidad se desplegaron transectos por triplicado de $100 \mathrm{~m}$ y $2 \mathrm{~m}$ a cada lado, abarcando un área de $400 \mathrm{~m}^{2}$ por transecto durante tres temporadas climáticas: de lluvia (noviembre-febrero), seca (marzo-junio) y de frentes fríos, también conocida localmente cómo época de nortes (juliooctubre) (De Jesús-Navarrete, Oliva-Rivera, Medina-Quej, \& Domínguez-Viveros, 1999).

Para la medición de las variables abióticas de temperatura $\left({ }^{\circ} \mathrm{C}\right)$ y salinidad (unidades prácticas de salinidad, ups) fueron colocados termógrafos marca HOBO (temperatura \& light data logger) programados para lectura cada $30 \mathrm{~min}$. El promedio de los datos obtenidos arrojó el valor medio mensual registrado para la zona de estudio.

Para el análisis de la densidad se utilizó el método de toma de muestras a distancia (Distance Sampling v. 6.0) de Buckland, Anderson, Burnhamk, y Laake (1993) considerando la distancia perpendicular de cada ejemplar al transecto y usando la función normal y coseno como serie de expansión. Se determinó la densidad total y de individuos adultos por zonas de estudio y temporadas (ind./ha, Media \pm DE). Los caracoles fueron recolectados y se les midió la longitud sifonal de la concha (Lsi) y el grosor del labio (Gl) para lo que se utilizó un calibrador de $0.01 \mathrm{~mm}$ de precisión. Los individuos, fueron clasificados de acuerdo al tamaño mínimo de captura legal en México (200mm de Lsi) (De Jesús Navarrete et al. 2003): en caracoles aptos para la pesca (Lsi $\geq 200 \mathrm{~mm}$ ) y caracoles no aptos para la pesca (Lsi<200mm); así como, en juveniles y adultos tomando como indicador de la madurez sexual el engrosamiento del 


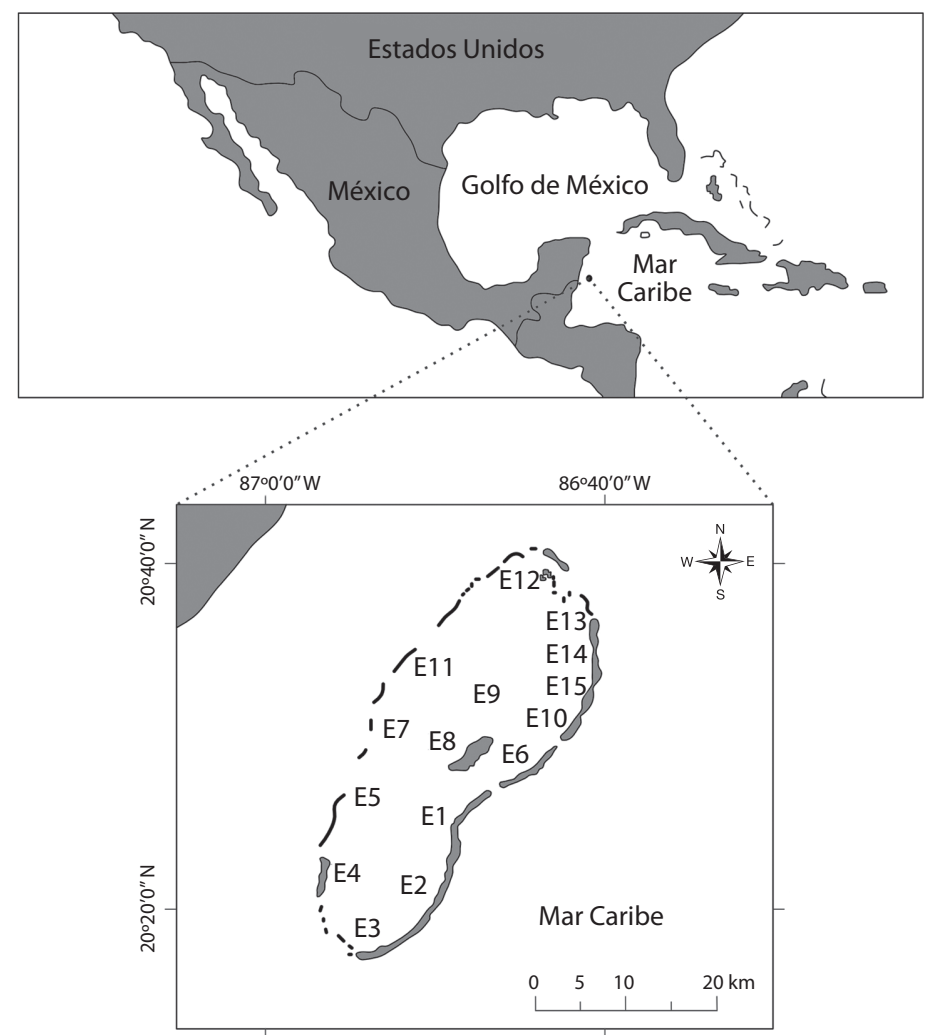

Fig. 1. Banco Chinchorro y sitios de muestreo (E1...E15).

Fig. 1. Banco Chinchorro and sampling sites (E1 ...E15).

labio. El margen de variación de la medida de grosor de labio que determina la edad de primera madurez y el inicio de la fase adulta es muy variable (Gómez Campo et al. 2010) pudiendo ser de $>1 \mathrm{~mm}$, utilizado por Pérez-Pérez y Aldana-Aranda (2003), hasta $14.2 \mathrm{~mm}$, registrado por Appeldoorn (1988), como promedio para el primer grupo de edad de individuos. Para este estudio se tuvo en cuenta la presencia de un labio extendido y el $\mathrm{Gl}<15.0 \mathrm{~mm}$ fueron clasificados como juveniles y con labio $\geq 15.0 \mathrm{~mm}$ como adultos (Stoner, Mueller, Brown, Davis \& Booker, 2012b).

En cada transecto se registró la frecuencia absoluta de las siguientes evidencias reproductivas: a) cópula: pene dentro del canal ovígero de la hembra, b) desove: hembras colocando la masa de huevo, c) agregaciones: caracoles agrupados (más de cinco individuos) y d) masas de huevo: masas de huevos libres (Pérez-Pérez \& Aldana-Aranda, 2003 con modificaciones). La frecuencia absoluta de las evidencias de reproducción se analizó en función de la densidad de individuos adultos por transecto, transformándolas a frecuencia relativa a partir del uso de la siguiente fórmula para cada zona y temporada de estudio:

$$
\text { frec. rel. }=\frac{\text { frec. abs. }}{\# \text { adultos }} \times 100
$$

Análisis de datos: Para evaluar diferencias en la densidad total y densidad de individuos adultos entre zonas y temporadas se aplicó un análisis de varianza (ANOVA) bifactorial, utilizando las zonas y temporadas como factores. Las diferencias entre las variables abióticas, también entre zonas y temporadas se abordaron a partir de análisis de varianza unifactoriales. En ambos casos, los datos fueron 
transformados a $\log (\mathrm{x}+1)$ con la finalidad de cumplir con el criterio de homocedasticidad. Se aplicaron pruebas de múltiples rangos $a$ posteriori en los casos necesarios para discernir diferencias identificadas en el ANOVA. La relación entre las densidades y las variables físicas se evaluó con un análisis de correlación de Pearson.

No fue posible evaluar la relación entre las evidencias reproductivas y las variables abióticas ya que las primeras fueron casi nulas en el área de estudio. El grado de asociación entre la Lsi y el Gl de los caracoles se calculó por el coeficiente de determinación $\left(\mathrm{r}^{2}\right)$. Las pruebas estadísticas fueron realizadas con el paquete SigmaPlot v. 11.0 y consideradas con un nivel de significancia del 95\% (Zar, 1999).

\section{RESULTADOS}

La temperatura del agua varió entre $24.5^{\circ} \mathrm{C}$ y $29.8^{\circ} \mathrm{C}$ durante el período de muestreo. No se observaron diferencias estadísticamente significativas en el comportamiento de las temperaturas entre las zonas de estudio (ANOVA, $\mathrm{p}>0.05$ ) pero sí entre los meses de las temporadas climáticas $(\mathrm{F}=126.1$, g.l. $=11, \mathrm{p}=0.001)$. La salinidad no mostró variación sustancial (entre 35.0 y 38.5 ups). No hubo diferencias significativas en la salinidad ni entre zonas (ANOVA, $\mathrm{p}>0.05)$ ni entre temporadas climáticas (ANOVA, $\mathrm{p}>0.05$ ). No se obtuvo correlación entre la densidad de caracoles (adultos y total) y los parámetros físicos (Pearson, $\mathrm{p}>0.05$ ).

Se muestreó un área total de $51600 \mathrm{~m}^{2}(129$ transectos). La densidad total para el área de estudio varió de 127ind./ha en época de lluvias a 384ind./ha en la época de secas (Fig. 2A). Espacialmente, la menor densidad se registró en la zona Norte con 198ind./ha y la mayor, en la zona Sur con 385ind./ha (Fig. 2B). En esta zona la densidad de adultos fue casi nula y solo se registraron valores de 6.11 ind./ha en época de seca y 8.33 ind./ha en la de lluvias. Por zonas de estudio, se registraron valores de 5.55, 7.05 y 8.33ind./ha para Centro, Sur y Norte, respectivamente. No se manifestaron diferencias significativas entre las temporadas de estudio en la densidad total (ANOVA, $\mathrm{p}>0.05$ ), ni tampoco en la densidad de adultos (ANOVA, $\mathrm{p}>0.05$ ). En el análisis espacial no se registraron diferencias significativas en cuanto a la densidad de adultos entre las zonas de estudio (ANOVA, $\mathrm{p}>0.05$ ). Sin embargo, sí se observaron diferencias significativas para la densidad total de caracoles entre las zonas de estudio ( $\mathrm{F}=17.2138$, $\mathrm{p}=0.0001$ ) (Cuadro 1). Una prueba de múltiples rangos realizada a posteriori permitió diferenciar la zona Sur de las zonas Centro y Norte, y entre estas dos últimas no existen diferencias (Fisher's LSD: $\mathrm{p}>0.05$ ).

Se recolectaron y midieron 1607 conchas durante el período de estudio. Las tallas variaron de 41 a $314 \mathrm{~mm}$ de Lsi. Los caracoles con Lsi mayor de $200 \mathrm{~mm}$, constituyeron el $42.1 \%$ de la población, predominando las tallas entre los 150 y $200 \mathrm{~mm}$ (58.8\%). El gráfico de frecuencias de tallas muestra dos picos claramente identificados el primero para las tallas entre 90 y $120 \mathrm{~mm}$ de Lsi, con una media de $76 \pm 31.5 \mathrm{~mm}$ y el segundo para las tallas entre 180 y $220 \mathrm{~mm}$ de Lsi, con una media de 194.25 $\pm 28.19 \mathrm{~mm}$ (Fig. 3).

\section{CUADRO 1}

Anova de dos vías para la densidad total de caracoles por temporadas y zonas de estudio en Banco Chinchorro

TABLE 1

Two ways ANOVA between conch total density, season and zone at Banco Chinchorro

\begin{tabular}{lccccc} 
Fuente de variación & Suma de cuadrados & Grados de libertad & Cuadrados medios & F & p \\
Zonas & 17722.40 & 2 & 8746.17 & 17.2138 & $* * 0.0001$ \\
Temp. & 1609.64 & 2 & 794.38 & 1.7690 & 0.1935 \\
Zonas x Temp. & 675.71 & 4 & 153.57 & 0.4369 & 0.7314 \\
Error & 32317.36 & 72 & 478.10 & & \\
\hline
\end{tabular}




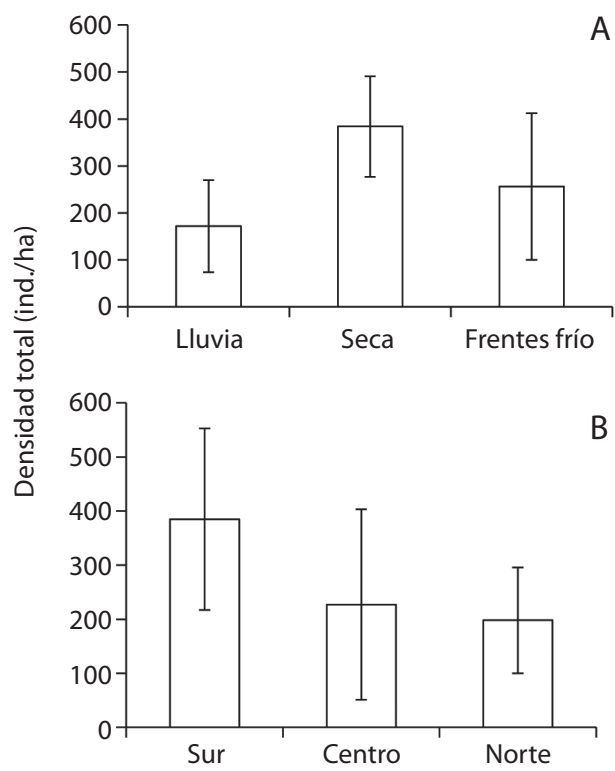

Fig. 2. Densidad media total de caracol rosado (barras verticales indican \pm desviación estándar) por temporadas climáticas (A) y zonas de estudio (B).

Fig. 2. Mean total densities of conch (vertical bars indicate \pm standard deviation) for climatic seasons (A) and study zone (B).

El análisis de la cantidad de caracoles adultos por temporada y zonas de estudio (Cuadro 2) mostró que $42 \%$ de la población de caracol de Banco Chinchorro puede ser vulnerable a la pesca por alcanzar la talla mínima de longitud sifonal autorizada para México (De Jesús
Navarrete et al. 2003). Sin embargo, el análisis de la madurez sexual tomando como indicador el grosor del labio (Stoner et al. 2012b) (Fig. 4), reflejó que sólo $2.2 \%$ de la población alcanza un $\mathrm{Gl} \geq 15 \mathrm{~mm}$, y se observó cómo la mayoría de los caracoles sobrepasan los 200mm de Lsi sin alcanzar al menos $10 \mathrm{~mm}$ de Gl.

La actividad reproductiva fue muy escasa. Sólo se observaron seis evidencias reproductivas durante todo el estudio. La mayor frecuencia de eventos reproductivos por temporada de muestreo y de acuerdo con la densidad de adultos se observó en la época seca (32\%), seguida por la de frentes fríos (28\%) y la lluvias (26\%) (Cuadro 3). En la zona Sur fue posible ver la mayor cantidad de evidencias reproductivas (27\%). No se observaron masas de huevos ni hembras desovando durante el muestreo; sólo se registraron dos cópulas, una en julio 2009 en la zona Sur, en un sitio de arena con parches de coral poco profunda $(3 \mathrm{~m})$ y una en abril 2010 también en una zona poco profunda $(2 \mathrm{~m})$ con fondo arenoso en la zona Centro. Durante el muestreo sólo se registraron cuatro agrupaciones de más de cinco individuos.

\section{DISCUSIÓN}

Las variaciones en la densidad de poblaciones de E. gigas han sido atribuidas fundamentalmente a fluctuaciones en las condiciones

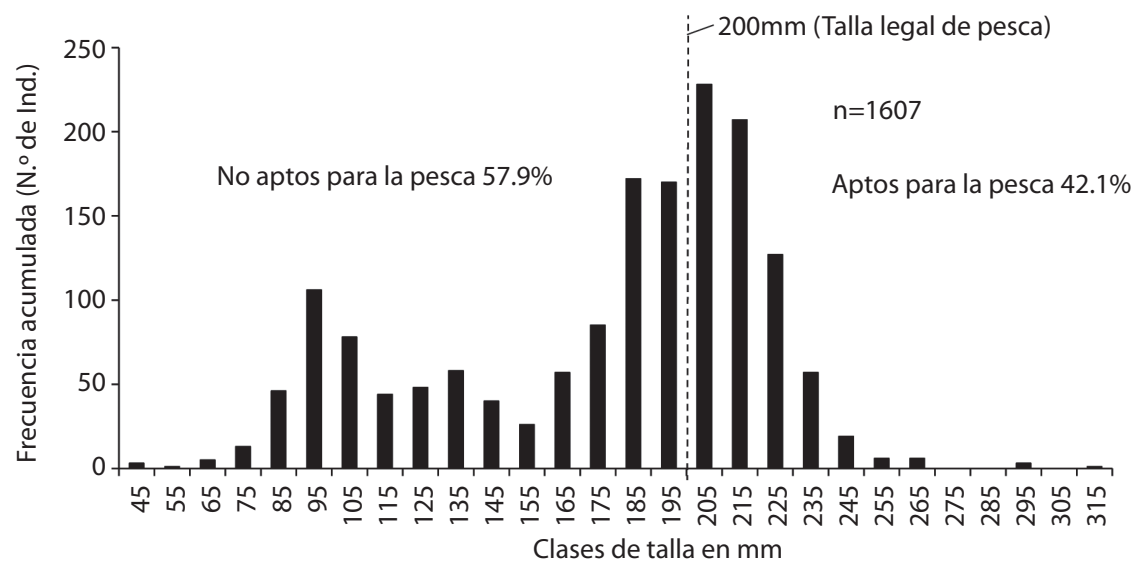

Fig. 3. Frecuencia de tallas para Banco Chinchorro durante la temporada 2009-2010.

Fig. 3. Size frequencies plot for Banco Chinchorro during the 2009-2010 seasons. 
CUADRO 2

Análisis comparativo entre el porcentaje de caracoles adultos y juveniles por zona y temporada de estudio tomando en consideración Lsi (200mm) talla de captura legal y Gl (15mm) indicador de la madurez sexual (Stoner et al. 2012b)

TABLE 2

Comparative analysis between percentage of adult and juvenile conchs by zone and study season and taking in consideration Lsi $(200 \mathrm{~mm})$ legal size of catch and $\mathrm{Gl}(15 \mathrm{~mm})$ as sexual maturity indicator (Stoner et al. 2012b)

\begin{tabular}{|c|c|c|c|c|c|c|c|}
\hline & \multirow{2}{*}{$\mathrm{N}$} & \multicolumn{2}{|c|}{ Lsi (200mm) } & \multicolumn{2}{|c|}{ Gl $(15 \mathrm{~mm})$} & \multirow{2}{*}{ Total (n) } & \multirow{2}{*}{$\begin{array}{l}\text { Densidad } \\
\text { (ind./ha) }\end{array}$} \\
\hline & & Juv. (\%) & Adultos (\%) & Juv. (\%) & Adultos (\%) & & \\
\hline \multicolumn{8}{|l|}{ Temporada } \\
\hline Lluvia & 39 & 41.2 & 58.8 & 97.3 & 2.7 & 455 & $172 \pm 17.9$ \\
\hline Seca & 45 & 69.2 & 30.8 & 98.7 & 1.3 & 685 & $384 \pm 29.6$ \\
\hline Frente fríos & 45 & 63.3 & 36.7 & 97.4 & 2.6 & 467 & $256 \pm 37.8$ \\
\hline Total & 129 & 57.9 & 42.1 & 97.8 & 2.2 & 1607 & \\
\hline \multicolumn{8}{|l|}{ Zona } \\
\hline Sur & 39 & 42.1 & 57.9 & 99.2 & 0.8 & 867 & $385 \pm 38$ \\
\hline Centro & 45 & 55.2 & 44.8 & 98.8 & 1.2 & 487 & $227 \pm 57.2$ \\
\hline Norte & 45 & 76.4 & 23.6 & 95.4 & 4.6 & 253 & $198 \pm 61$ \\
\hline Total & 129 & 57.9 & 42.1 & 97.8 & 2.2 & 1607 & \\
\hline
\end{tabular}

\section{CUADRO 3}

Frecuencia absoluta y relativa (\%) de las evidencias reproductivas por zona y temporada de estudio en Banco Chinchorro durante la temporada 2009-2010

TABLE 3

Absolute and relative frequency (\%) of reproductive evidences by zone and study season at Banco Chinchorro during 2009-2010 seasons

\begin{tabular}{|c|c|c|c|c|c|c|c|c|}
\hline & \multirow{2}{*}{$\mathrm{N}$} & \multirow{2}{*}{ Total } & \multicolumn{4}{|c|}{ Frecuencia absoluta y relativa (\%) } & \multirow{2}{*}{$\begin{array}{c}\text { Densidad } \\
\text { total(ind./ha) }\end{array}$} & \multirow{2}{*}{$\begin{array}{l}\text { Densidad adultos } \\
\text { (ind./ha, } \mathrm{Gl} \geq 15 \mathrm{~mm} \text { ) }\end{array}$} \\
\hline & & & Cópula & Desove & Masas de huevo & Agregaciones & & \\
\hline \multicolumn{9}{|l|}{ Temporada } \\
\hline Frente fríos & 45 & $2(28)$ & $1(14)$ & 0 & 0 & $1(14)$ & $256 \pm 37.8$ & $6.67 \pm 116.3$ \\
\hline Seca & 45 & $2(32)$ & $1(16)$ & 0 & 0 & $1(16)$ & $384 \pm 29.6$ & $6.11 \pm 101.5$ \\
\hline Lluvia & 39 & $2(26)$ & 0 & 0 & 0 & $2(26)$ & $172 \pm 17.9$ & $8.33 \pm 87.9$ \\
\hline \multicolumn{9}{|l|}{ Zona } \\
\hline Norte & 45 & $1(7)$ & 0 & 0 & 0 & $1(7)$ & $198 \pm 61$ & $8.33 \pm 90.7$ \\
\hline Centro & 45 & $2(20)$ & $1(10)$ & 0 & 0 & $1(10)$ & $227 \pm 57.2$ & $5.55 \pm 107$ \\
\hline Sur & 39 & $3(27)$ & $1(9)$ & 0 & 0 & $2(18)$ & $385 \pm 38$ & $7.05 \pm 108.2$ \\
\hline Total & 129 & $6(17)$ & $2(6)$ & 0 & 0 & $4(11)$ & $311.4 \pm 47.3$ & $6.98 \pm 112$ \\
\hline
\end{tabular}

ambientales, interacciones biológicas, variaciones en el reclutamiento y a la pesca como acción modificadora del medio (Weil \& Laughling, 1984; Rouhgarden, Ganes \& Possingham, 1988; De Jesús-Navarrete et al. 2003, GómezCampo, Rueda, García-Valencia, Ballesteros \& Mejía, 2005).

Este trabajo muestra que las densidades resultaron inferiores a las de estudios anteriores en Banco Chinchorro (De Jesús-Navarrete,
2001; De Jesús-Navarrete \& Valencia-Beltrán, 2003; De Jesús-Navarrete et al. 2003; MedinaQuej et al. 2005; Basurto-Origel et al. 2007). La evidencia demostró que no sólo las densidades totales están disminuyendo sino también las densidades de caracoles adultos.

Se reportan alteraciones en la densidad y tasas de crecimiento en poblaciones de bacalao (Gadus morhua) en el este de Canadá (Swain, Sinclair, \& Hanson, 2007) y se han atribuido 




Fig. 4. Relación entre longitud sifonal y grosor del labio para la población de caracol en Banco Chinchorro, México. (Cuadrante I contiene caracoles maduros son $\mathrm{Gl} \geq 15 \mathrm{~mm}$, cuadrante II contiene caracoles aptos para la pesca $\mathrm{Lsi} \geq 200 \mathrm{~mm}$ pero que no alcanzan $\operatorname{los} 15 \mathrm{~mm}$ de Gl, línea discontinua indica $\mathrm{Gl}=15 \mathrm{~mm}$, puntos discontinuos indican $\mathrm{Lsi}=200 \mathrm{~mm}$.

Fig. 4. Siphonal length and lip thickness relationship for queen conch population at Banco Chinchorro, Mexico. (quadrant I contains maturity conchs with $\mathrm{Gl} \geq 15 \mathrm{~mm}$, quadrant II contains conchs suitable for fishing Lsi $\geq 200 \mathrm{~mm}$ but that no reach $15 \mathrm{~mm}$ of $\mathrm{Gl}$, discontinuous line indicates $\mathrm{Gl}=15 \mathrm{~mm}$, discontinuous points indicates $\mathrm{Lsi}=200 \mathrm{~mm}$.

esos cambios a la presión selectiva de la pesca. Jorgensen et al. (2007) concluyen que los efectos evolutivos ocasionados por la presión pesquera pueden ser generalizados y constituyen una preocupación importante en la gestión de las pesquerías. Por otra parte, la sobrepesca se ha asociado con la disminución de E. gigas en otras partes del Caribe (Appeldoorn, 1994b) con reducción de la longitud de los caracoles y las densidades en lugares importantes de su distribución como Belice en 1979, causando una disminución en la captura comercial (Brownell \& Stevely, 1981).

El problema de la sobrepesca del caracol en Banco Chinchorro ha sido abordado en estudios precedentes (De Jesús-Navarrete et al. 2003), mostrando una disminución de la densidad de población desde 1990 a 1997. Además, estos autores mencionan que la población de caracol en Banco Chinchorro en esos años estuvo dominada por individuos jóvenes (más del $89 \%$ ) y sugieren que tanto la pesca legal como ilegal fueron los responsables de estos cambios, a pesar de los esfuerzos de gestión por parte de la Comisión Nacional de Pesca (CONAPESCA) para regular la pesquería.

La población de caracol de Banco Chinchorro durante este estudio estuvo compuesta principalmente de caracoles jóvenes cuya Lsi ronda los $200 \mathrm{~mm}$, pero que no alcanzan la madurez sexual. Los caracoles aptos para la pesca representaron el $42 \%$ de la población tomando como criterio la talla mínima de captura. Sin embargo, el análisis del grosor del labio como indicador de la madurez sexual refleja que solo cerca del $2 \%$ de la población es de adultos maduros. Resultados similares ya había sido reportados por De Jesús-Navarrete et al. (2003) quienes encontraron en su estudio que menos del 5\% de la población eran caracoles adultos. Estos autores alertaron que si la densidad continuaba disminuyendo podía verse un efecto negativo sobre la reproducción en un corto plazo, como efectivamente está ocurriendo en la actualidad.

Evidentemente, la sobrepesca ya sea legal o ilegal, ha de ser la causa principal de la disminución de la población de caracol en el Banco Chinchorro. Esto es debido fundamentalmente a que los pescadores capturan el número de caracoles necesario para completar la cuota asignada para cada año, lo que resulta en una disminución de la población de adultos y la consiguiente disminución de la tasa de encuentro entre hembras y machos, lo que sugiere la presencia de un efecto Allee en la población. Este problema se acrecienta con los pescadores furtivos que extraen los caracoles sin importar el tamaño y aún no existe el marco legal apropiado para evitarlo. Contrariamente a la reproducción de E. gigas reportadas durante todo el año para Banco Chinchorro (Cruz, 1986; Corral \& Ogawa, 1987), sólo se observaron dos cópulas durante el estudio, lo que podría resultar de una reducción en la densidad de adultos.

La disminución en la densidad de adultos con el cambio de criterios para evaluar la madurez sexual, es una prueba de los errores en la gestión de la pesca de caracol en Banco Chinchorro durante más de dos décadas y puede ser la causa de la disminución de la 
población y el colapso de la pesquería en el Banco Chinchorro y el resto del Mar Caribe (Stoner et al. 2012b).

Para las Bahamas, se ha reportado que en poblaciones con densidades menores de 56ind/ha no son posibles la cópula ni el desove (Stoner \& Ray, 2000). Nuestros resultados indican que las densidades de caracol adulto en Banco Chinchorro están muy por debajo de este umbral mínimo necesario. Esto podría explicar la baja actividad reproductiva en el área de estudio.

Las diferencias encontradas entre las zonas de muestreo podrían explicarse por los movimientos locales del E. gigas a lo largo y ancho de la laguna arrecifal. Estos movimientos locales se relacionan fundamentalmente con la migración ontogenética de los caracoles hacia las zonas profundas y luego a zonas someras en donde tiene lugar la reproducción (Laughlin \& Weil, 1985, Díaz-Avalos, 1991). Por otro lado, se conoce que existe una estratificación del recurso respecto a la profundidad y que los individuos se encuentran en grupos de tamaños y edad similares (Randall, 1964; Alcolado, 1976), esta estratificación ha sido reportada por Domínguez-Viveros, Sosa-Cordero \& MedinaQuej (1999) para Banco Chinchorro, destacando el papel de la profundidad en la distribución de los organismos. Estos autores señalan que en general, la zona Sur del Banco Chinchorro presenta una profundidad mayor que las zonas Norte y Centro.

La profundidad mayor en la zona Sur del Banco Chinchorro puede brindar refugio a los caracoles que moviéndose hacia estas zonas quedan fuera del alcance de los pescadores. Las características del hábitat también podrían estar influyendo en la distribución de la especie dentro del banco ya que las zonas Norte y Centro han sido reportadas como zonas de crianza y desarrollo de juveniles, así como la zona Sur como zona de agregaciones de adultos debido a la calidad del hábitat (Domínguez-Viveros et al. 1999).

Las evidencias reproductivas fueron casi nulas en el área de estudio. Este resultado está relacionado directamente con la densidad total y densidad de adultos en cada zona. Appeldoorn (1988) propuso tres posibles mecanismos para explicar la relación entre la densidad y el éxito reproductivo, todos ellos relacionados con la densidad de adultos reproductores, mientras que Stoner et al. (2012a) mencionan la necesidad de mantener una densidad mayor que 100ind./ha para asegurar completamente la reproducción. Aunque estos parámetros pueden variar geográficamente, las bajas densidades de adultos aquí reportados pueden ser la causa de la baja actividad reproductiva, lo que pone en evidencia que el efecto Allee está actuando en la población de caracol de Banco Chinchorro y que es absolutamente necesario el cierre total de su pesquería para recuperar la población.

Consideraciones de manejo: Nuestros resultados muestran evidencia de que el efecto Allee puede estar actuando sobre la población de caracol de Banco Chinchorro debido a las condiciones de sobrepesca a las que se ve sometida.

Las estrategias de manejo para las pesquerías del E. gigas deben centrarse en capturar sólo el exceso de la producción o incluso, establecer una cuota por debajo de este exceso, teniendo en cuenta las necesidades locales, y manteniendo un nivel de abundancia en la población, donde se mantenga la tasa de encuentro por encima del límite inferior necesario para la reproducción (Appeldoorn, 1988; Dugan \& Davis, 1993; Basurto et al., 2000; Stoner et al. 2012a). Esto evita el desequilibrio de la población, manteniendo la biomasa global y evitando la disminución en el tamaño y la madurez sexual (Harmelin, Bachet \& García, 1995). Además, promueve el mantenimiento de una tasa de reclutamiento anual, evitando la consiguiente reducción de la fertilidad debido a la baja tasa de encuentro entre parejas reproductoras (efecto Allee).

Según estos principios, es necesario mantener una densidad óptima en la población entre 56ind./ha y 100ind./ha (Stoner et al. 2012a). Para lograr estas densidades en Banco Chinchorro, es necesario tomar en consideración los siguientes aspectos: 
- Cierre total de la pesquería (una veda permanente por cinco años que contribuya a la recuperación de la población).

- Monitoreo sistemático: la implementación de un programa de monitoreo sistemático debe ser incluida en el plan de manejo del área protegida, solo así es posible evaluar el comportamiento de la población en el tiempo y valorar si la veda está siendo efectiva.

- Refugios de pesca: el establecimiento de al menos dos refugios de pesca para el caracol en Banco Chinchorro puede ser una estrategia exitosa, ya que involucra dos objetivos concretos: la protección de la población reproductora una vez levantada la veda y también la pesca sostenible del recurso fuera del refugio.

Los refugios de pesca para el caracol pueden ser diseñados e implementados por los pescadores. La densidad de caracoles adultos en el refugio puede ser regulada a partir del movimiento de caracoles adultos a los refugios por los pescadores; y puede ser evaluada y protegida una vez aprobados los refugios en el plan de manejo del área. La densidad óptima dentro del refugio debe ser mayor de 100ind./ ha y una proporción de sexos de 1:1; de esta forma, se podría asegurar la producción de larvas constante y el efecto derrame de estas hacia zonas circundantes (Guénette, Lauck \& Clark, 1998; Micheli, Saenz-Arroyo, Greenley, Vázquez \& Espinoza-Montes, 2012; Varkey, Ainsworth \& Pitcher, 2012).

\section{AGRADECIMIENTOS}

Agradecemos el apoyo del Consejo Nacional de Ciencia y Tecnología, México, concesión doctoral número 228610. La asistencia en campo del equipo de trabajo de la Reserva de Biosfera Banco Chinchorro, en especial a G. Muñoz-Salcedo, así como el apoyo de la CONANP y SEMARNAT para las salidas de campo. Y. Ventura-Días por su colaboración en la elaboración de los mapas. Los comentarios de P.M. Alcolado y M. Mendoza contribuyeron a enriquecer el manuscrito.

\section{RESUMEN}

Eustrombus gigas es uno de los recursos pesqueros costeros más valiosos en la región del Caribe y constituye un molusco con un elevado valor estético y ecológico. En esta investigación se evalúa su densidad total, densidad de adultos, la estructura de tallas y frecuencia de evidencias reproductivas en Banco Chinchorro, México, periodo 2009-2010; durante tres períodos (de lluvias, seca y de frentes fríos) en tres zonas: Norte, Centro y Sur. La densidad total para el área de estudio varió de 127ind./ha en lluvias a 384ind./ha en seca, la menor densidad se presentó en la zona Norte del Banco (198ind./ha) y la mayor en la zona Sur (385ind./ha). La densidad de caracoles adultos fue baja (6.11ind./ha en seca y 8.33 ind./ha en lluvias y 5.55, 7.05 y 8.33 ind./ha para las zonas Centro, Sur y Norte, respectivamente). Las densidades de adultos estuvieron por debajo del umbral mínimo necesario para mantener la reproducción. El $42 \%$ de la población es vulnerable a la pesca (Lsi $\geq 200 \mathrm{~mm}$ ); sin embargo, solo $2.2 \%$ de la población alcanza un Gl $>15 \mathrm{~mm}$. Solo se observaron seis evidencias de reproducción durante el período de estudio, lo que señala la presencia del efecto Allee en la población y la urgente necesidad del cierre de su pesquería. Se proponen tres puntos importantes para el manejo de la especie: cierre de la pesca, monitoreo de la población y la implementación refugios pesqueros.

Palabras clave: efecto Allee, sobrepesca, reproducción, áreas protegidas, Eustrombus gigas.

\section{REFERENCIAS}

Abbott, R. T. (1974). American seashells. Nueva York, USA: Van Nostrand Reinhold.

Alcolado, P. M. (1976). Growth, morphological variations of the shell and some biological data of the conch (Cobo) Strombus gigas L. (Mollusca, Mesogastropoda). Serie Oceanológica, 34, 1-36.

Aldana-Aranda, D., Baqueiro-Cárdenas, E., MartínezMorales, I., \& Ochoa-Báez, I. (2003). Gonad behavior during peak reproduction period of Strombus gigas from Banco Chinchorro. Bulletin of Marine Science, 73, 241-248.

Appeldoorn, R. S. (1988). Ontogenetic changes in natural mortality rate of queen conch. Strombus gigas, (Mollusca: Mesogastropoda). Bulletin of Marine Science, 42, 159-165.

Appeldoorn, R. S. (1990). Growth of juvenile queen conch Strombus gigas L., of La Parguera, Puerto Rico. Journal of Shellfish Research, 9, 59-62. 
Appeldoorn, R. S. (1993). Reproduction, spawning potential ratio and larval abundance of queen conch of $L a$ Parguera, Puerto Rico (Ms. Rept.). San Juan, Puerto Rico: Caribbean Fishery Management Council.

Appeldoorn, R. S. (1994a). Queen conch management and research: status, needs and priorities. In R. S. Appeldoorn, \& B. Rodriguez (Eds.), Queen Conch Biology, fisheries and mariculture: (pp. 301-319). Caracas, Venezuela: Fundación Científica Los Roques.

Appeldoorn, R. S. (1994b). Spatial variability in the morphology of Queen Conch and its implication for management regulations. In R. S. Appeldoorn, \& B. Rodriguez (Eds.), Queen Conch Biology, fisheries and mariculture (pp. 145-157). Caracas, Venezuela: Fundación Científica Los Roques.

Ávila-Poveda, O. H., \& Baqueiro-Cárdenas, E. R. (2006). Size at sexual maturity in the queen conch Strombus gigas from Colombia. Boletín de Investigaciones Marinas y Costeras, 35, 223-233.

Basurto-Origel, M., Cruz-Domínguez, O., Martínez-Vázquez, D., \& Cadena-Romero, P. (2000). Caracol. In M. A Cisneros-Mata, L. F. Beléndez-Moreno, E. Zárate-Becerra, M. T. Gaspar-Dillanes, L. LópezGonzález, C. Saucedo-Ruíz, \& J. Tovar-Ávila (Eds.), Sustentabilidad y pesca responsable en México; evaluación y manejo (pp. 712-744). México D.F. México: INP-SAGARPA.

Basurto-Origel, M., Cadena, P., Escobedo, G., \& Fernández, F. (2005). Evaluación de la población de Strombus gigas en los bancos de Cozumel y Chinchorro y recomendaciones para su aprovechamiento sostenible. Quintana Roo, México: Instituto Nacional de la Pesca.

Basurto-Origel, M., Cadena, P., Escobedo, G., \& Figueroa, F. (2007). Evaluación de la población de Strombus gigas en los bancos abiertos a la pesca en Quintana Roo, México. Banco Chinchorro y Banco de Cozumel. Temporada de captura 2007-2008. Quintana Roo, México: Instituto Nacional de la Pesca.

Basurto-Origel, M., Espinosa, J. C., Cob, E., Escobedo, G., \& Figueroa, F. (2009). Análisis de biomasa de caracol rosado Strombus gigas en Banco Chinchorro. Estimación de cuota de captura para la temporada de pesca 2009-2010. Quintana Roo, México: Instituto Nacional de la Pesca.

Basurto-Origel, M., Espinosa, J. C., Cob, E., \& Escobedo, G. (2010). Evaluación de la abundancia de Strombus gigas en Banco Chinchorro y cálculo de la cuota de pesca para la temporada de pesca 2010-2011. Quintana Roo, México: Instituto Nacional de la Pesca.

Basurto-Origel, M., Cervera, K., \& Medina, M. (2011). Evaluación de la abundancia de Strombus gigas en Banco Chinchorro y cálculo de la cuota de pesca para la temporada de pesca 2011-2012. Quintana Roo, México: Instituto Nacional de la Pesca.
Brownell, W. N., \& Stevely, J. M. (1981). The biology, fisheries, and management of the queen conch, Strombus gigas. Marine Fisheries Review, 43, 1-12.

Buckland, S. T., Anderson, D. R., Burnham, K. P., \& Laake, J. L. (1993). Distance sampling estimating abundance of biological populations. London, England: Chapman \& Hall.

Chávez-Villegas, J. F., Enríquez-Díaz, M., Cid-Becerra, J. A., \& Aldana-Aranda, D. (2012). Abundancia y distribución de larvas de Strombus gigas (Mesograstropoda: Strombidae) durante el periodo reproductivo de la especie en el Caribe Mexicano. Revista de Biología Tropical, 60, 89-97.

Corral, J. L., \& Ogawa, J. (1987). Cultivo masivo de larvas de caracol Strombus gigas en estanques de concreto. Proceedings of the Gulf and Caribbean Fisheries Institute 38, 345-351.

Cruz, R. (1986). Avances en la experimentación de producción masiva de caracol en Quintana Roo, México. Proceedings of the Gulf and Caribbean Fisheries Institute 37, 12-20.

De Jesús-Navarrete, A., \& Oliva-Rivera, J. (1997). Densidad, crecimiento y reclutamiento del caracol rosado Strombus gigas L. (Gasterópoda: Strombidae) en Quintana Roo, México. Revista de Biología Tropical, 45, 797-801.

De Jesús-Navarrete, A. (1999). Abundancia de larvas velígeras del caracol rosado Strombus gigas y su relación con las áreas de pesca en el Sur de Quintana Roo, México. (Informe técnico final Proyecto 0420PN). Chetumal, Quintana Roo, México: El Colegio de la Frontera Sur.

De Jesús-Navarrete, A., Oliva-Rivera, J., Medina-Quej, A., \& Domínguez-Viveros, M. (1999). Growth, recruitment and population structure of the pink conch (Strombus gigas) in Punta Gavilán, Quintana Roo, Mexico. Proceedings of the Gulf and Caribbean Fisheries Institute 46, 74-83.

De Jesús-Navarrete, A. (2001). Crecimiento del Caracol Strombus gigas (Gastropoda: Strombidae) en cuatro ambientes de Quintana Roo, México. Revista de Biología Tropical, 49, 85-91.

De Jesús-Navarrete, A., Medina-Quej, A., \& Oliva-Rivera, J. (2003). Changes in the queen conch (Strombus gigas L.) population structure at Banco Chinchorro, Quintana Roo, Mexico, 1990-1997. Bulletin of Marine Science, 73, 219-229.

De Jesús-Navarrete, A., \& Valencia-Beltrán, V. (2003). Abundance of Strombus gigas zero-year class juveniles at Banco Chinchorro Biosphere Reserve, Quintana Roo, Mexico. Bulletin of Marine Science, 73, 231-240.

Díaz-Avalos, C. (1991). Growth and mortality of juvenile queen conch Strombus gigas in Punta Gavilán, 
Quintana Roo. Some considerations on its fishery utilization. Ciencia Pesquera, 8, 63-70.

Domínguez-Viveros, M., Sosa-Cordero, E., \& MedinaQuej, A. (1999). Abundance and population parameters of queen conch Strombus gigas in Banco Chinchorro, Quintana Roo, Mexico. Proceedings of the Gulf and Caribbean Fisheries Institute, 45, 943-954.

Dugan, J. E., \& Davis, G. E. (1993). Applications of marine refuge to coastal fisheries management. Journal of Fisheries and Aquatic Sciences, 50, 2029-2042.

Egan, D. B. (1985). Aspects of the reproductive biology of Strombus gigas. (Unpublished master Thesis). The University of British Columbia, USA.

Glazer, R., \& Berg Jr., C. J. (1992). Growth and mortality of the queen conch, Strombus gigas, in Florida, a progress report. Proceedings of the Gulf and Caribbean Fisheries Institute, 42, 153-157.

Gómez-Campo, K., Rueda, M., García-Valencia, C., Ballesteros, F., \& Mejía, L. E. (2005). Densidad y distribución de la estructura poblacional de Strombus gigas Linnaeus, 1758 (Mollusca: Strombidae) asociada a diferentes hábitats en el Archipiélago Nuestra Señora del Rosario, Caribe colombiano. Proceedings of the Gulf and Caribbean Fisheries Institute, 58, 399-406.

Gómez-Campo, K., Rueda, M., \& García-Valencia, C. (2010). Distribución espacial, abundancia y relación con características del hábitat del caracol Pala Eustrombus gigas (Linnaeus) (Mollusca: Strombidae) en el archipiélago Nuestra Señora del Rosario, Caribe Colombiano. Boletín de Investigaciones Marinas y Costeras, 39, 137-159.

Guénette, S., Lauck, T., \& Clark, C. (1998). Marine reserves: from Beverton and Holt to the present. Review in Fish Biology and Fisheries, 8, 251-272.

Harmelin, J. G., Bachet, F., \& García, F. (1995). Mediterranean marine reserves: fish indices as a test of protection efficiency. Marine Ecology, 16, 233-250.

INE-SEMARNAP. (2000). Programa de Manejo de la Biosfera Banco Chinchorro, México. México D.F. México: Comisión Nacional de Áreas Naturales Protegidas.

Jared, M., Taylor, M., Roy, K., \& Hellberg, M. (2006). A molecular phylogenetic analysis of strombid gastropod morphological diversity. Molecular Phylogenetic Evololution, 41, 436-434.

Jorgensen, C., Enberg, K., Dunlop, E. S., Arlinghaus, R., Boukal, D. S., Brander, K., \& Rijnsdorp, A. D. (2007). Ecology: managing evolving fish stocks. Science, 318, 1247-1248.

Laughlin, R. A., \& Weil, E. (1985). Ecology, cultivation, and repopulation of the queen conch Strombus gigas L. in the National Park of Los Roques Archipiélago
(Tech. Rept. 1182).Caracas, Venezuela: Fundación Científica Los Roques.

Medina-Quej, A., Domínguez-Viveros, M., \& de JesúsNavarrete, A. (2005). Relaciones morfométricas en el caracol rosado Strombus gigas en Banco Chinchorro, Quintana Roo, México. Proceedings of the Gulf and Caribbean Fisheries Institute, 47, 811-823.

Micheli, F., Saenz-Arroyo, A., Greenley, A., Vázquez, L., \& Espinoza-Montes, J. A. (2012). Evidence That Marine Reserves Enhance Resilience to Climatic Impacts. PLoS ONE, 7, 1-8.

Paris, C. B., Aldana-Aranda, D., Pérez-Pérez, M., \& Kool, J. (2008). Connectivity of Queen Conch, Strombus gigas, populations from Mexico. Proceedings of the Coral Reef Symposium 11, 439-443.

Peel, J. R., \& Aldana-Aranda, D. (2012). Growth and population assessment of the queen conch Strombus gigas (Mesogastropoda: Strombidae) by capture markrecapture sampling in a natural protected area of the Mexican Caribbean. Revista de Biología Tropical, 60, 127-137.

Pérez-Pérez, M., \& Aldana-Aranda, D. (2003). Actividad reproductiva de Strombus gigas (Mesogasteropoda: Strombidae) en diferentes hábitats del Arrecife Alacranes, Yucatán. Revista de Biología Tropical, 51, 119-126.

Pérez-Enríquez, R., García-Rodríguez, F. J., MendozaCarrión, G., \& Padilla, C. (2011). Geographical variation in the genetic diversity and composition of the endangered Queen Conch Strombus gigas (Mesograstrópoda: Strombidae) from Yucatan, Mexico. Revista de Biología Tropical, 59, 1115-1126.

Petuch, E. J. (2004). Cenozoic Seas. The View from Eastern North America. Florida, USA: CRC, Boca Ratón.

Petuch, E. J., \& Roberts C. E. (2007). The Geology of the Everglades and Adjacent Areas. Florida, USA: CRC, Boca Ratón.

Randall, J. E. (1964). Contributions to the biology of the queen conch, Strombus gigas. Bulletin of Marine Science, 14, 246-295.

Rouhgarden, J., Ganes, S., \& Possingham, H. (1988). Recruitment dynamics in complex life cycles. Science, 228, 1460-1466.

Stoner, A. W., Sandt, V. J., \& Boidron-Metairon, I. F. (1992).Seasonality in reproductive activity and larval abundance of queen conch Strombus gigas. Fisheries Bulletin, 90, 161-170.

Stoner, A. W., Ray, M., Glazer, R., \& McCarthy, K. J. (1996). Metamorphic responses to natural substrata in a gastropod larva: decisions related to post-larval growth and habitat preference. Journal of Experimental Marine Biology and Ecology, 205, 229-243.

Stoner, A. W., \& Ray, M. (2000). Evidence for Allee effects in an over-harvested marine gastropod: 
density-dependent copulation and egg production. Marine Ecology Progress Series, 202, 297-302.

Stoner, A. W., Davis, M. H., \& Booker, C. J. (2012a). Negative consequences of Allee effect are compounded by fishing pressure: comparison of queen conch reproduction in fishing grounds and a marine protected area. Bulletin of Marine Science, 88, 89-102.

Stoner, A. W., Mueller, K. W., Brown, N. J., Davis, M. H., \& Booker, C. J. ( 2012b). Maturation and age in queen conch (Strombus gigas): urgent need for changes in harvest criteria. Fisheries Research, 131133, 76-84.

Swain, D. P., Sinclair, A. F., \& Hanson, J. M. (2007). Evolutionary response to size-selective mortality in an exploited fish population.Proceedings of the Royal Society of Biology and Biological Sciences, 274, 1015-1022.

Theile, S. (2001). Queen conch fisheries and their management in the Caribbean. Brussels, Belgium: TRAFFIC Europe, CITES.
Varkey, D., Ainsworth, C. H., \& Pitcher, T. J. (2012). Modeling Reef Fish Population Responses to Fisheries Restrictions in Marine Protected Areas in the Coral Triangle. Journal of Marine Biology, 12, 1-8.

Vázquez-Yeomans, L., Ordóñez-López, U., Quintal-Lizama, C., \& Ornelas-Roa, M. (2003). A preliminary fish larvae survey in Banco Chinchorro. Bulletin of Marine Science, 73, 141-152.

Vokes, H. E., \& Vokes, E. H. (1983). Distribution of Shallow-Water Marine Mollusca, Yucatán Peninsula, México (Monograph 1, Publication 54). Mexico D.F, Mexico: Mesoamerican Ecology Institute.

Weil, M. E., \& Laughlin, R. (1984). Biology, population dynamics, and reproduction of the queen conch Strombus gigas Linné in the Archipiélago de Los Roques National Park. Journal of Shellfish Research, 4, 45-62.

Zar, J. H. (1999). Biostatistical Analysis. Upper Saddle River, USA: Prentice-Hall. 
